\title{
Robotically Controlled Directivity and Gain Measurements of Integrated Antennas at $280 \mathrm{GHz}$
}

Linus Boehm, Sebastian Pledl, Frank Boegelsack, Martin Hitzler, Christian Waldschmidt resale or redistribution to servers or lists, or reuse of any copyrighted component of this work in other works. 


\title{
Robotically Controlled Directivity and Gain Measurements of Integrated Antennas at $280 \mathrm{GHz}$
}

\author{
Linus Boehm, Sebastian Pledl, Frank Boegelsack, Martin Hitzler, Christian Waldschmidt \\ Institute of Microwave Techniques \\ University of Ulm \\ Ulm, Germany \\ forename.surname@uni-ulm.de
}

\begin{abstract}
In this paper a detailed description of an automated mm-wave antenna measurement setup is given, and gain and directivity measurement results are shown for a standard gain horn antenna and an integrated antenna at $280 \mathrm{GHz}$. The setup comprises a vector network analyzer (VNA) with frequency converters for different frequency bands, a six degree of freedom industrial robot, and a controller that provides the interface and controls the measurements. This setup makes it possible to measure gain, directivity, radiated power, and efficiency of both waveguide fed and integrated chip antennas at frequencies between $60 \mathrm{GHz}$ and $330 \mathrm{GHz}$ with high accuracy. Furthermore, the setup is highly configurable. Arbitrary trajectories on arbitrary planes around the AUT (antenna under test) can be measured quickly without having to change any hardware.
\end{abstract}

\section{INTRODUCTION}

Using mm-wave frequencies for distance and speed sensors allows to build integrated radar sensors. Especially when using cheap CMOS technology, the production costs compared to radars at lower frequencies can be reduced significantly thus allowing for low-cost mass market radar sensors. As the antennas provide the link between chip and environment, the antennas are a crucial part for the overall performance of the radar chip, which is why high gain antennas with high radiation efficiencies are needed. The characterization of antennas at frequencies above $100 \mathrm{GHz}$ requires elaborate measurement setups in order to achieve accurate results despite the small wavelength.

At lower frequencies the AUT is often turned to measure the directivity with a static reference antenna. Setups for accurate measurements of integrated antennas must allow to contact the antenna with a waver prober, which means that the AUT cannot be moved during the measurement. Therefore the reference antenna has to be moved around the AUT [1]. Setups that allow integrated antenna measurements are often costummade and restricted in their performance and reconfigurability. The measurement scenarios are often limited to a specific planar or spherical measurement type, the correct alignment of AUT and Rx antenna is complicated, e.g. [2]. The presented system overcomes these problems and provides the possibility to measure antenna gain, radiated power, directivity, and radiation pattern. A 6 degrees of freedom industrial robot is used for easy, fast, and precise measurements. In order to be able to measure not only waveguide fed, but also integrated

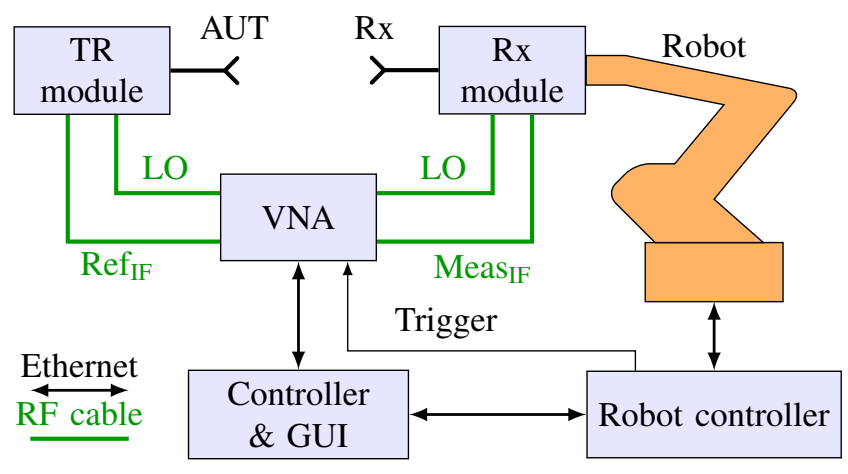

Fig. 1. Schematic of the measurement setup.

antennas a probe station is part of the system to allow chip contact.

\section{OVERVIEW}

A block diagram of the setup is shown in Fig. 11. The controller provides a graphical user interface (GUI), where different measurement scenarios, frequency ranges, resolutions, maximum speed, and acceleration and other measurement parameters can be chosen. After a measurement was initiated, the required parameters are sent over Ethernet to the robot and the VNA. Both on-the-fly (OTF) and point-to-point (PTP) measurements are supported. For OTF measurements, the robot will follow the selected trajectory with constant speed, allowing for fast measurements with minimal acceleration and therefore minimizing vibrations. If multiple frequencies were selected, the measurement procedure is conducted once for every frequency. As the robot moves, it will trigger the VNA measurements at the desired locations. The robot will keep moving with constant speed during a measurement.

During a PTP measurement the robot stops at every measurement position. After reaching a measurement location, the robot waits a specified time to let vibrations abate that were caused by the deceleration. Then the robot triggers the VNA and the measurement is performed. Once it is completed the robot moves to the next position. PTP measurements need more time than OTF measurements, but for measurements with multiple frequencies, the robot does not need to go through 


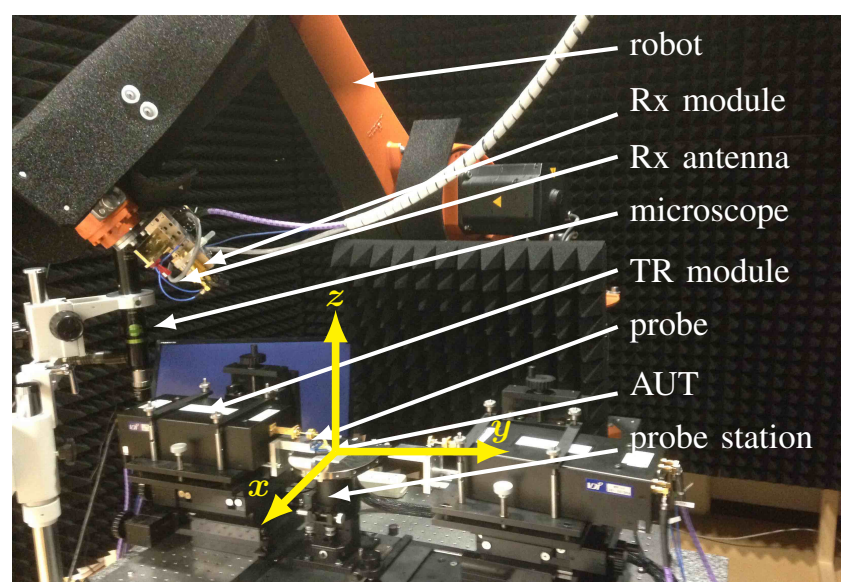

Fig. 2. Measurement setup with robot, converter modules, and probe station.

the entire course multiple times and the measuring position is more accurate.

\section{COMPONENTS}

\section{A. Robot}

The Rx module with the Rx antenna is mounted to the robot. The robot moves around the AUT on the desired trajectory. Planar scans can be realized by movements in the $x$-y-plane at a certain distance to the AUT. Far field measurements are performed by moving the Rx antenna on a sphere around the AUT. For all azimuth movements, the Rx antenna is turned around its axis in order to maintain the correct polarization during the entire measurement.

The measurement angle for spherical far field measurements strongly depends on the desired radius. For radii of $30 \mathrm{~cm}, 3 \mathrm{D}$ measurements with angles up to $|\theta| \approx 45^{\circ}$ (see Fig. 4) are realizable. For smaller radii, the maximum angle increases. The maximum angle for spherical measurements is limited by the movements in the $y$ - $z$-plane. In this plane the movement is hampered by the robot itself in $-x$-direction and by the arm length in $+x$-direction (see coordinate system in Fig. 2). In the $y$ - $z$-plane bigger radii and maximum angles $|\theta|>90^{\circ}$ are possible. An additional rotator as described in [3] could be used to turn the AUT in $\phi$ direction to make use of the higher flexibility in the $y$-z-plane, however, this is not feasible when using a probe station to contact integrated antennas.

Near field measurements can be performed on areas bigger than $1 \mathrm{~m}^{2}$, but the phase accuracy of the setup has to be further investigated for meaningful near field measurements.

\section{B. Measurement Equipment}

A vector network analyzer (VNA) with a frequency range from $10 \mathrm{MHz}$ to $67 \mathrm{GHz}$ is used for the measurements. Frequency converters for Rx and Tx allow to extend the frequency range to $330 \mathrm{GHz}$. The converter modules can be seen in Fig. 2. The TR converter can both transmit and receive signals and is used as a transmitter to feed the AUT. The Rx converter

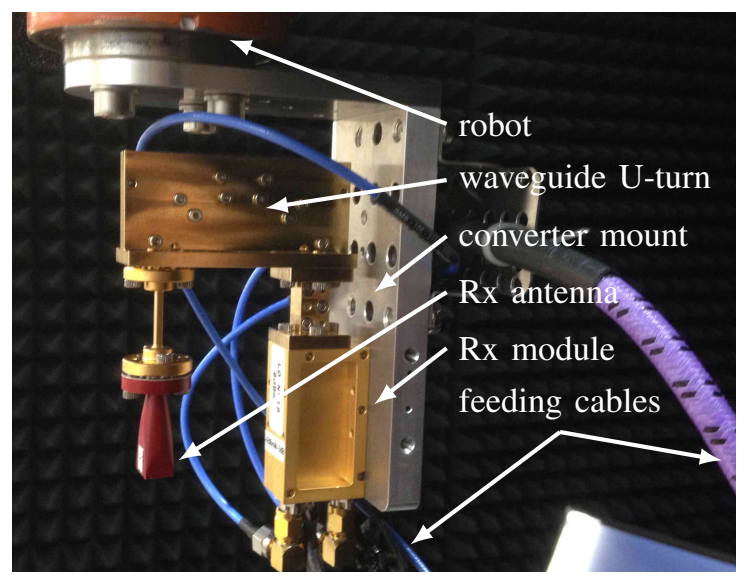

Fig. 3. Rx converter mount with reference antenna.

module is used for down conversion of the received signal. As this module must be mounted to the robot flange, it was optimized for minimum size and can only receive, but not transmit any signals. A detailed picture of the converter and the mounting structure is shown in Fig. 3

\section{Probe Station}

The probe station offers high reconfigurability as both the chuck and the towers holding the probes, and converter modules can be placed anywhere on the station. This allows for multiple probe configurations. The probes can be placed such that they face each other or they can be installed at a $90^{\circ}$ angle, which makes it possible to have various pad configurations without causing any contacting issues. Furthermore, the influence of probes close to antennas for different probe orientations and positions relative to the AUT can be investigated.

\section{Control Computer}

The control computer provides a GUI for simple interaction with the setup. Through the GUI the Rx antenna dimensions can be entered, the Rx antenna can be aligned with the AUT, different measurement scenarios (OTF, PTP, near field, far field) and their respective settings can be chosen.

\section{Measurement Procedure}

In order to perform a measurement, Rx antenna and AUT have to be aligned. First, the relative position of the Rx antenna to the hand of the robot have to be entered by specifying the dimensions $(x, y, z)$ as well as the relative rotation around the three axis $\left(\operatorname{Rot}_{\mathrm{x}}, \operatorname{Rot}_{\mathrm{y}}, \operatorname{Rot}_{\mathrm{z}}\right)$. Once the system knows the $\mathrm{Rx}$ dimensions, the position of the AUT must be specified, which can be done with a laser range finder that is attached to the robot. By centering the device over the AUT, the $x$ - and $y$ position of the AUT is taught. When the antennas are aligned, the relative $z$-position can be measured with the range finder. After the alignment the origin of the measurement will be in the center of the AUT. The polarization of the measurement can be adjusted by rotating the $\mathrm{Rx}$ antenna around the $z$-axis. 


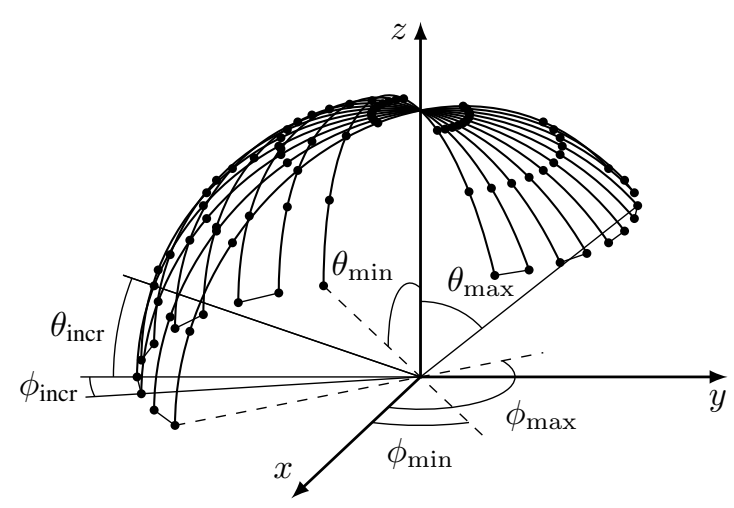

Fig. 4. Far field measurement trajectory.

After the alignment process, the measurement parameters can be chosen. For spherical measurements these parameters are the measurement speed, acceleration, distance, averaging factor as well as minimum, maximum, and increment for frequency, $\phi$, and $\theta$. The maximum measurement speed was set to $40^{\circ} / \mathrm{s}$, the maximum acceleration to $100^{\circ} / \mathrm{s}^{2}$.

The number of cuts and their orientation is specified by $\phi_{\min }, \phi_{\max }$, and $\phi_{\text {incr }}$ as shown in Fig. 4. The dimensions of each cut and the number of measurement points on a cut is specified by $\theta_{\min }, \theta_{\max }$, and $\theta_{\text {incr }}$.

\section{Calibration}

For the gain measurement in Section VIII the ratio of the transmitted power $P_{t}$ and the received power $P_{r}$ is required. As the reference planes are at the two ports of the VNA, the measured $S_{21 \text {,meas }}$ is the cascading of the transmitter, the free space propagation, and the receiver:

$$
\left.S_{21, \text { meas }}\right|_{\mathrm{dB}}=\left.S_{21, \mathrm{TR}}\right|_{\mathrm{dB}}+10 \log _{10}\left(\frac{P_{r}}{P_{t}}\right) \mathrm{dB}+\left.S_{21, \mathrm{Rx}}\right|_{\mathrm{dB}},
$$

where $S_{21, \mathrm{TR}}$ and $S_{21, \mathrm{Rx}}$ are the transmission factors of the transmitter and the receiver. $\left.S_{21, \mathrm{TR}}\right|_{\mathrm{dB}}+\left.S_{21, \mathrm{Rx}}\right|_{\mathrm{dB}}$ can be measured by connecting transmitter and receiver back to back and $\frac{P_{r}}{P_{t}}$ can then be calculated.

An additional calibration was necessary for the integrated antenna, as a probe was used to contact the antenna. The gain of the probe was determined with the TRL deembedding technique [4].

\section{DYNAMIC RANGE}

The dynamic range is set by the minimum and maximum AUT gain that can be measured. The lower boundary for the gain is when the received signal is equal to the noise floor. For lower gains the received signal would be below the noise floor and therefore not measurable. For a of $R$ the minimum gain for which a signal can be detected is

$$
G_{t, \min }=\frac{P_{\mathrm{r}, \text { noise }}}{P_{t}}\left(\frac{4 \pi R}{\lambda}\right)^{2} \frac{1}{G_{r}} .
$$

The upper limit for the dynamic range is set by the maximum power accepted at the input of the Rx module. At the same

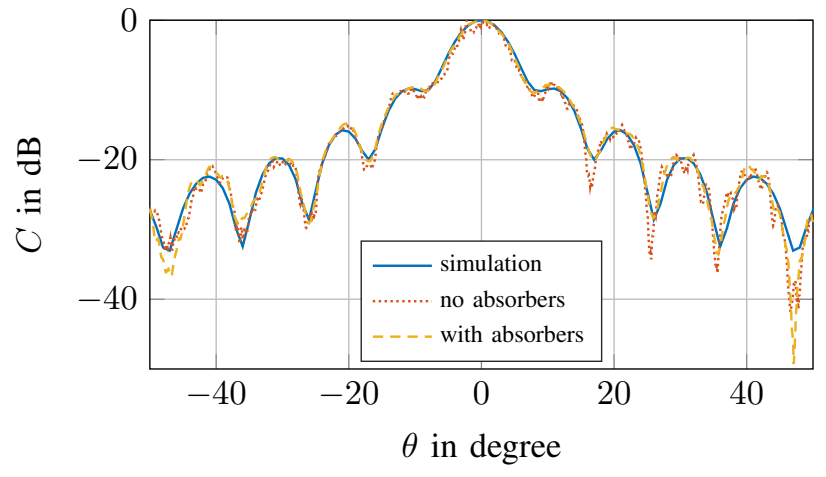

Fig. 5. E-plane simulation and measurement at $275 \mathrm{GHz}$.

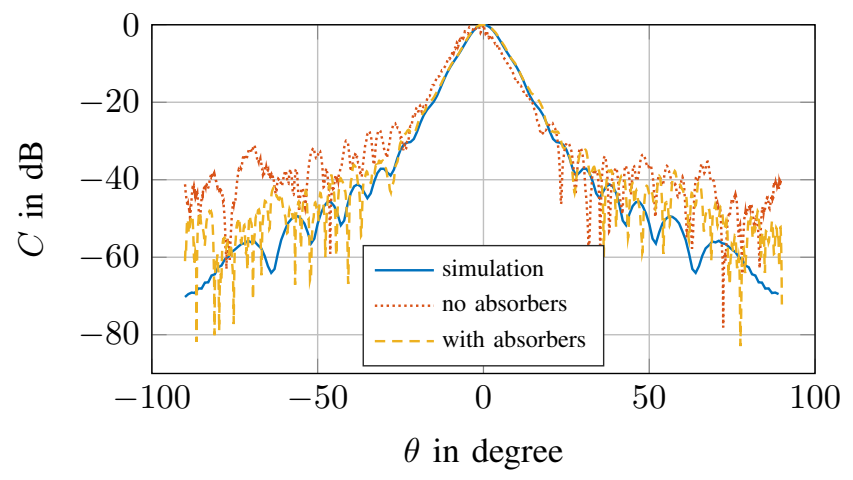

Fig. 6. H-plane simulation and measurement at $275 \mathrm{GHz}$.

distance $R$ and for the same transmit power $P_{t}$, the maximum measurable gain is therefore

$$
G_{t, \max }=\frac{P_{\mathrm{r}, \max }}{P_{t}}\left(\frac{4 \pi R}{\lambda}\right)^{2} \frac{1}{G_{r}} .
$$

The dynamic range is

$$
\frac{G_{t, \max }}{G_{t, \min }}=\frac{P_{\max }}{P_{\text {noise }}} .
$$

The maximum allowed power at the input port of the $\mathrm{Rx}$ converter is $0 \mathrm{dBm}$. The noise level depends on the averaging factor and the IF-bandwidth. For an IF-bandwidth of $100 \mathrm{~Hz}$ and without averaging the noise floor is at $-100 \mathrm{dBm}$ and thus the dynamic range would be $100 \mathrm{~dB}$ in this case.

\section{Measurement Results And Absorber INFLUENCE}

In order to assess the accuracy of the setup a standard gain horn antenna was measured in the E-plane and H-plane at a distance of $350 \mathrm{~mm}$ and with an elevation resolution of $1^{\circ}$. The minimum far field distance is $\frac{2 D^{2}}{\lambda}=220 \mathrm{~mm}$, where $D$ is the biggest antenna dimension. The measured directivity was compared to an EM simulation of the antenna in Fig. 5 and 6 for both E- and H-plane respectively. The dotted lines are measurements without absorbers, which leads to measurement inaccuracies due to reflections at the probe station and the robot. The main source of reflections is the probe station. 


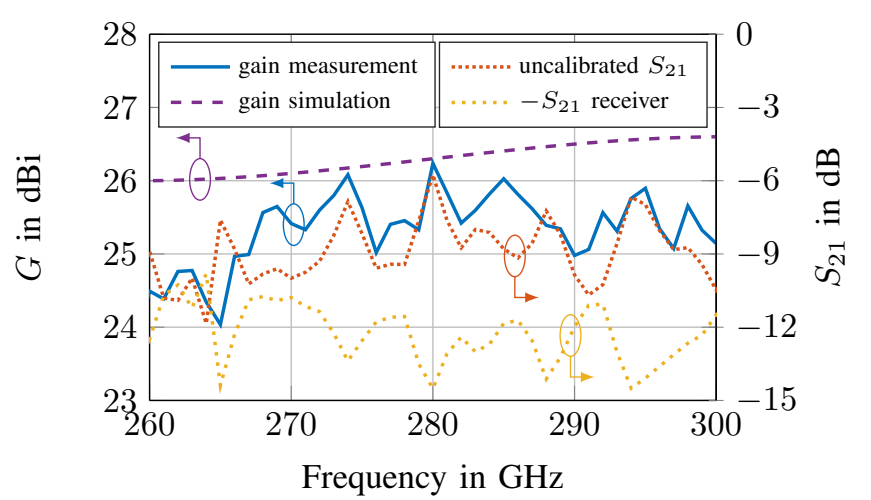

Fig. 7. Gain of a horn antenna from $260 \mathrm{GHz}$ to $300 \mathrm{GHz}$.

When absorbers are used to shield the robot and the probe station, simulation and measurement are very similar regarding both the amplitude and the location of sidelobes and poles up to a signal level of around $-40 \mathrm{dBm}$.

\section{GAIN MEASUREMENT}

The gain of a standard horn antenna as a reference and the gain of an integrated antenna were measured. The integrated antenna was the antenna presented in [5], scaled to $280 \mathrm{GHz}$. The gain of the standard gain horn antenna was measured using the Friis equation with known $G_{r}$ and $P_{t}$. The integrated antenna was measured with a reference horn antenna.

\section{A. Absolute Gain Measurement}

After the calibration procedure described in Section $\mathrm{V}, \frac{P_{r}}{P_{t}}$ is known and therefore the gain can be calculated using the Friis formula. In Fig. 7 the measured (solid) and simulated (dashed) gain of a horn antenna is shown from $260 \mathrm{GHz}$ to $300 \mathrm{GHz}$. The simulated gain was $26.3 \mathrm{~dB}$ at $280 \mathrm{GHz}$. The ripple of the measured gain is due to imperfect calibration. The loosely dotted line shows the measured $S_{21}$ of the system, which has a peak-to-peak value of $\approx 5 \mathrm{~dB}$. The uncalibrated measurement results (densely dotted) have the same ripple. Through the calibration the peak-to-peak difference was reduced to $\approx 2 \mathrm{~dB}$, but the effects of the non-ideal $\mathrm{Rx}$ module can still be seen clearly. The ripple is mainly caused by the receiver mounted to the robot. The output power of the TR module was $-11 \mathrm{dBm}$ and steady over the regarded frequency range.

\section{B. Reference Antenna}

Another option to measure the gain $G_{\mathrm{AUT}}$ of an unknown antenna is using a reference antenna with a well known gain $G_{\text {ref }}$. By measuring the $S_{21}$ for the reference antenna $S_{21 \text {,ref }}$ and the AUT $S_{21, \text { AUT }}$, the gain of the unknown antenna can be determined with

$$
\left.G_{\mathrm{AUT}}\right|_{\mathrm{dB}}=\left.S_{21, \mathrm{AUT}}\right|_{\mathrm{dB}}-\left.S_{21, \mathrm{ref}}\right|_{\mathrm{dB}}+\left.G_{\mathrm{ref}}\right|_{\mathrm{dB}}-\left.S_{21, \text { probe }}\right|_{\mathrm{dB}},
$$

where $S_{21 \text {,probe }}$ is the calculated gain of the probe.

The measured and simulated directivity of the AUT as well as a picture of the integrated antenna with the dielectric resonator at $280 \mathrm{GHz}$ is shown in Fig. 8. The attenuation for

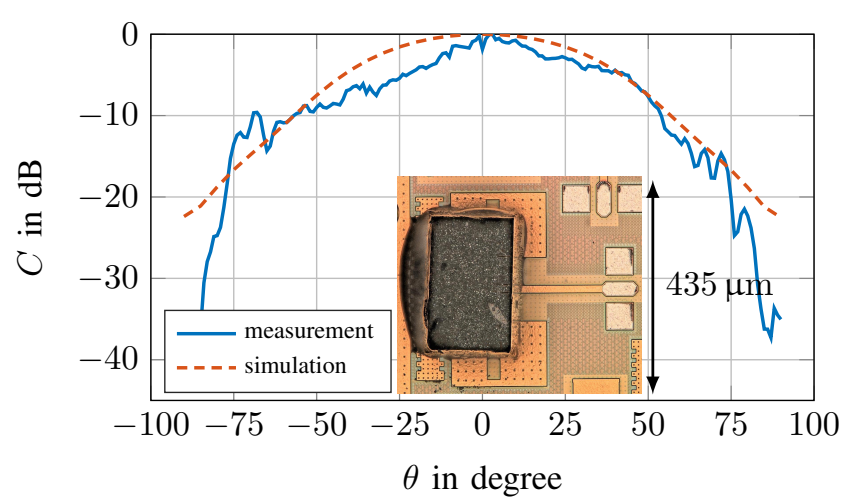

Fig. 8. Directivity measurement and simulation at $280 \mathrm{GHz}$.

angles over $80^{\circ}$ is caused by attenuators that were placed on the chuck to avoid reflections.

The measured $S_{21 \text {,AUT }}$ was $-28.2 \mathrm{~dB}$. With a reference antenna gain of $G_{\mathrm{ref}}=26.2 \mathrm{~dB}, S_{21, \text { ref }}=-5.8 \mathrm{~dB}$ was measured. The TRL deembedding of the probe resulted in $S_{21, \text { probe }}=-5 \mathrm{~dB}$. With $(5)$ this results in $G_{\mathrm{AUT}}=8.7 \mathrm{~dB}$. The simulated gain was $6.9 \mathrm{~dB}$. Possible error sources are the calibration of the receiver and losses of the substrate, which are not precisely known at $280 \mathrm{GHz}$.

\section{CONCLUSION}

In this paper a detailed description of an antenna measurement setup that offers high flexibility and the possibility to measure integrated antennas at frequencies up to $330 \mathrm{GHz}$ fast and reliably was given. The components were described and the measurement procedure was explained. Directivity measurement results were presented, which show high consistency with simulation results for both antennas. Lastly, gain measurements were performed of a standard gain horn antenna and an integrated antenna at $280 \mathrm{GHz}$. The measured gain is consistent with the simulated gain within the measurement tolerances. A possible approach to further improve measurement results is to reduce the effect of the receiver ripple over the frequency with an enhanced calibration procedure.

\section{REFERENCES}

[1] S. Beer and T. Zwick, "Probe Based Radiation Pattern Measurements For Highly Integrated Millimeter-Wave Antennas," in Antennas and Propagation (EuCAP), Proceedings of the Fourth European Conference on, pp. 1-5, 2010.

[2] Z.-M. Tsai, Y.-C. Wu, S.-Y. Chen, T. Lee, and H. Wang, "A V-Band On-Wafer Near-Field Antenna Measurement System Using an IC Probe Station," Antennas and Propagation, IEEE Transactions on, vol. 61, no. 4, pp. 2058-2067, 2013.

[3] D. Novotny, "Antenna Alignment and Positional Validation of a mmWave Antenna System Using 6D Coordinate Metrology," in Antennas and Propagation, pp. 1-6, Aug. 2014.

[4] G. F. Engen and C. A. Hoer, "Thru-Reflect-Line: An Improved Technique for Calibrating the Dual Six-Port Automatic Network Analyzer," Microwave Theory and Techniques, IEEE Transactions on, vol. 27, no. 12, pp. 987-993, 1979.

[5] D. Hou, W. Hong, W. L. Goh, J. Chen, Y.-Z. Xiong, S. Hu, and M. Madihian, "D-Band On-Chip Higher-Order-Mode Dielectric-Resonator Antennas fed by Half-Mode Cavity in CMOS Technology," Antennas and Propagation Magazine, IEEE, vol. 56, no. 3, pp. 80-89, 2014. 